\title{
Tipología familiar y dinámica bullying/ciberbullying en Educación Secundaria
}

\author{
Virginia Palacios García, Ma Isabel Polo del Río, Elena Felipe Castaño, \\ Benito León del Barco y Fernando Fajardo Bullón \\ Universidad de Extremadura (España)
}

Las situaciones de bullying y ciberbullying son, tristemente, acontecimientos cada vez más presentes en la comunidad escolar, especialmente favorecidas, en el caso del ciberbullying, por el gran auge de las redes sociales que se está produciendo en nuestra sociedad. Los factores que intentan explicar estas situaciones de violencia entre iguales han puesto en evidencia aspectos de los individuos involucrados en ellas, pero no debemos olvidar otras variables que, como la tipología familiar, son fundamentales en esta etapa del ciclo vital y por tanto, pueden jugar también un papel importante. El objetivo del presente trabajo es analizar si la pertenencia a algunos de los diferentes grupos familiares existentes en nuestra sociedad podía relacionarse con la incidencia de situaciones de bullying y de ciberbullying. Los participantes fueron 1.684 alumnos pertenecientes a 22 centros de Secundaria (IES e IESO) de la Comunidad Autónoma de Extremadura siendo el 50.9\% de la muestra mujeres y el $49.1 \%$ varones. Los resultados obtenidos permiten afirmar que, existen más víctimas y agresores/as entre familias no nucleares (familias monoparentales, familias reconstituidas, crianza por parte de los abuelos,...) que entre familias nucleares, sin embargo esta relación no se ha encontrado entre los observadores.

Palabras clave: Tipología familiar, bullying, ciberbullying, participantes bullying, acoso escolar.

Family typology and dynamic bullying/cyberbullying in Secondary Education. The situations of bullying and cyberbullying are, sadly, increasingly present events in the school community, especially favored in the case of cyberbullying, by the boom of social networking taking place in our society. The factors that attempt to explain these peer violence situations have revealed aspects of the individuals involved in them, but we must not forget other variables, such as family type, are crucial at this stage of the life cycle and thus may play also an important role. The aim of this paper is to analyze whether belonging to some of the different family groups existing in our society could be related to the incidence of bullying situations and cyberbullying. Participants were 1684 students from 22 secondary schools (IES and IESO) of the Autonomous Community of Extremadura being $50.9 \%$ of the sample women and $49.1 \%$ men. The results confirm that there are more victims and aggressors among non-nuclear families (single parent families, blended families, parenting by grandparents,...) than nuclear families, however this relationship was not found among observers.

Key words: Family typology, bullying, cyberbullying, participants bullying.

Correspondencia: Virginia Palacios García. Departamento de Psicología y Antropología. Universidad de Extremadura. Avda. de la Universidad, s/n. C.P. 10001. Cáceres (España). E-mail: virgynyapg@gmail.com 
La unidad familiar no es tan sólo una estructura formada por un conjunto de personas unidas por lazos de parentesco y reforzados por una convivencia, sino también una forma de vivir en intimidad y de compartir responsabilidades económicas, sociales y emocionales. Desde un punto de vista psicológico, la familia se considera como una red de comunicaciones entrelazadas en la que participan todos sus miembros siendo el primer núcleo de socialización. La familia ha experimentado importantes transformaciones a lo largo de la historia, las cuales han propiciado que emerjan diferentes tipos de familias, algunos de ellos aparecen recogidos en la figura 1.

Figura 1. Principales tipos de familias

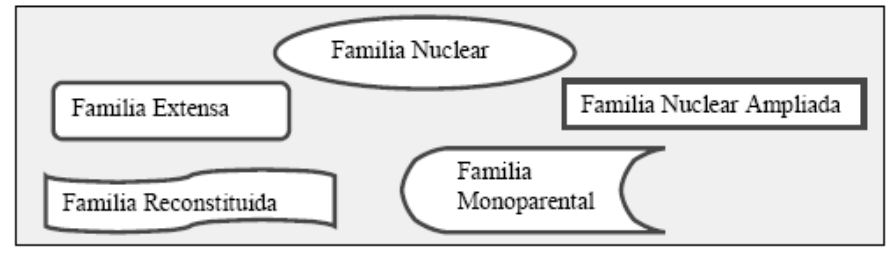

La pervivencia de la familia a lo largo de los años se ha visto favorecida debido a que ésta cumple importantes funciones para el desarrollo de los individuos. Por un lado, asume funciones internas como la protección biológica, psicológica y social, y por otro lado, se encarga de transmisión de valores culturales facilitando la socialización de sus miembros. Todas ellas facilitan el desarrollo de diversas competencias en los/as menores, especialmente en la competencia social, donde puede ser distinguidas una serie de variables parentales que afectan al desarrollo social de los/as menores como:

- Las relaciones vinculares con los padres.

- Las ideas, creencias y percepciones de los padres sobre el desarrollo y la educación de sus hijos/as.

- Los estilos educativos familiares (democrático, negligente, autoritario y permisivo) cuyas consecuencias no se limitan a los años de la infancia, sino que se prolongan en la adolescencia y la juventud (Baumrind, 1991).

Por tanto, las competenciales sociales de los/as menores guardan una estrecha relación con su conducta agresiva. Una de las conductas violentas que han adquirido una mayor prevalencia en las últimas décadas ha sido el acoso escolar o bullying, la cual se manifiesta entre escolares (Ahmad y Smith, 1990; Besag, 1989; Cerezo y Esteban, 1992; Floyd, 1989; Laslet, 1980; Lowenstein, 1977; Olweus, 1978). Olweus (1983) definió el bullying como una conducta de persecución física y/o psicológica que realiza un alumno/a contra otro, al que elige como víctima de repetidos ataques. En la actualidad las formas tradicionales de maltrato han ido modificándose de forma sustancial, 
apareciendo manifestaciones más específicas llamadas ciberbullying (Ortega, Calmaestra y Mora-Merchan, 2008; Smith, Mahdavi, Carvalho y Tippet, 2006). En general, se considera que quienes participan en el bullying son: quienes ejercen la intimidación directa o indirectamente (agresores), quienes la sufren directa o indirectamente (las víctimas) y quienes contemplan estos acontecimientos (los testigos) cuyas características aparecen reflejadas en la tabla 1 .

Tabla 1. Características de los participantes en situaciones de bullying

\begin{tabular}{cl}
\hline \multicolumn{1}{c}{ Rol } & \multicolumn{1}{c}{ Características } \\
& Características Personales: Impulsividad, agresividad, extroversión, diversos niveles de autoestima, \\
& necesidad de ejercer dominio sobre los demás, dificultades de autocontrol, falta de empatía, baja escala \\
Agresor & moral... \\
& Aspectos Físicos: Varones, mayor fortaleza física... \\
& Ámbito Social: Mala integración escolar, perfiles de conducta interpersonal dominantes... \\
\hline & Características Personales: Timidez, inseguridad, baja asertividad, alta ansiedad, autoconcepto negativo, \\
& pobres estrategias de afrontamiento... \\
& Aspectos Físicos: Varones, desviaciones externas, desequilibrio en la fuerza física... \\
& Ámbito Social y Relaciones Interpersonales: Menos habilidosos, bajos niveles de popularidad, \\
& retraimiento social, miedo a establecer nuevas relaciones, buena actitud con profesorado, buenas \\
& relaciones familiares...
\end{tabular}

El maltrato entre iguales es un fenómeno bastante complejo cuya explicación no puede atribuirse a una sola causa sino que debe asumirse una explicación ecológica, de forma que puede considerarse como el resultado de la interacción compleja entre factores del individuo y factores que surgen de los distintos contextos en los que la persona vive, desde los más próximos como la familia, la escuela, el grupo de amigos o los medios de comunicación; hasta los más lejanos como los recursos educativos, culturales y económicos.

Diversos estudios han dirigido su atención hacia la influencia de la familia en el fenómeno de bullying, ya que ésta conlleva un adecuado ajuste psicosocial en los/as menores, evitando así su implicación en comportamientos problemáticos (Dekovic, Wissink y Meijer, 2004; Martínez, Fuertes, Ramos y Hernández, 2003; Musitu, Buelga, Lila y Cava, 2001).

Sin embargo, no existen demasiados estudios que se hayan ocupado directamente del análisis que pueden ejercer diferentes variables mediadoras como la familia en las conductas violentas de los/as adolescentes, ya que la mayoría han explorado el papel desempeñado por otras variables mediadoras como son la actitud negativa de los/as menores ante la autoridad institucional o la baja autoestima (Molpeceres, Lucas y Pons, 2000; O’Moore, 1997), las cuales podrían tener su origen en el contexto familiar.

Con este trabajo se pretende analizar la relación entre la tipología familiar y la incidencia en situaciones de bullying y ciberbullying en estudiantes de Educación Secundaria. 


\section{MÉTODO}

\section{Participantes}

Han participado en el estudio un total de 1684 alumnos/as pertenecientes a 22 centros de Secundaria (IES e IESO) de la Comunidad Autónoma de Extremadura. La muestra estaba compuesta por 857 mujeres $(50.9 \%)$ y por 827 hombres $(49.1 \%)$. La distribución por género y nivel educativo de los participantes está recogida en la tabla 2 , y en la tabla 3 los estadísticos descriptivos referidos a la edad de los alumnos/as.

Tabla 2. Distribución del número de participantes según género y curso

\begin{tabular}{ccccc}
\hline \multirow{4}{*}{ Curso } & & \multicolumn{3}{c}{ Sexo } \\
\cline { 2 - 5 } & & Mujer & Hombre & Total \\
\hline \multirow{4}{*}{ Trimero ESO } & 213 & 248 & 461 \\
\cline { 2 - 5 } & Segundo ESO & 216 & 234 & 450 \\
\cline { 2 - 5 } & Tercero ESO & 222 & 171 & 393 \\
\cline { 2 - 5 } & Cuarto ESO & 206 & 174 & 380 \\
\hline Total & & 857 & 827 & 1684 \\
\hline
\end{tabular}

\section{Procedimiento}

La selección de los estudiantes se realizó mediante un muestreo polietápico estratificado por conglomerados y la selección aleatoria de los grupos en los centros que disponían de varias líneas en los cursos $1^{\circ}, 2^{\circ}, 3^{\circ}$ y $4^{\circ}$ de la ESO. Los estratos que se consideraron fueron los Centros de Recursos de Profesores (CPRs) que dividen en 18 zonas geográficas la Comunidad de Extremadura, cada CPR tiene asignados unos determinados Centros de Secundaria (IES e IESO). El muestreo por conglomerado consistió en seleccionar al azar varios centros de cada CPR. Algunos centros declinaron la participación en el estudio, por lo que varios CPRs no tuvieron representación.

El número de participantes se determinó a partir del número de alumnos matriculados en Secundaria en Extremadura durante el curso 2008-2009, considerando un error muestral de 3\% y un nivel de confianza de 95.5\%. La administración de los cuestionarios contaba con una duración aproximadamente de 30 minutos y se realizó de forma colectiva en grupos de unas 25 ó 30 personas. La participación de los sujetos fue voluntaria previo consentimiento informado de forma verbal, asegurándoles la confidencialidad de los datos y su uso exclusivo en investigación.

Las variables empleadas en este estudio son las siguientes:

Variable Independiente: Tipo de Familia dicotomizada en dos valores:

Familia Nuclear: Formada por los padres y sus hijos/as

Familia Extensa o no Nuclear: En ella se recogen diferentes núcleos familiares como pueden ser familias monoparentales, familias reconstituidas, crianza de los/as menores por parte de los abuelos/as,... 
Variables Dependientes:

Frecuencia de Observadores.

Frecuencia de Víctimas.

Frecuencia de Agresores.

Tabla 3. Estadísticos descriptivos relacionados con la edad

\begin{tabular}{cccc}
\hline Media & Desviación Típica & Mínimo & Máximo \\
\hline 14.24 & 1.365 & 18 & 11 \\
\hline
\end{tabular}

\section{Instrumentos}

Los instrumentos empleados en el estudio fueron los siguientes:

Cuestionario del Defensor del Pueblo-Unicef (2006). Es un cuestionario autoadministrado que analiza la incidencia del acoso escolar desde tres perspectivas: observador, agresor y víctima. Las preguntas incluyen trece conductas de acoso clasificadas en seis modalidades de maltrato. Su ocurrencia es evaluada en una escala ordinal de frecuencia con cuatro categorías y también, evalúa algunas características de los agresores como curso, género y los lugares en los que ocurre el acoso escolar.

Cuestionario Cyberbullying de Ortega, Calmaestra y Mora Merchán (2007). Este cuestionario está basado en el de Smith, Mahdavi, Carvalho y Tippet (2006), aunque ha sufrido una serie de modificaciones como una reducción del número de ítems, y se han añadido preguntas que hacen referencia a las vías por las que se ejerce el acoso, a la comparación del cyberbullying con el acoso tradicional, a los sentimientos que provocan las acciones de cyberbullying tanto en víctimas como agresores y a las estrategias de afrontamiento empleadas. Las preguntas abarcan dos modalidades sobre las situaciones de cyberbullying, en primer lugar aquellas en las que se emplea el teléfono móvil y en segundo lugar, las situaciones en las que se utiliza Internet.

\section{RESULTADOS}

En primer lugar, se llevó a cabo un análisis de frecuencias de la variable independiente Familia. Esta variable fue dicotomizada en dos grupos: Grupo 1 engloba la familia nuclear definida en el cuestionario a través de la respuesta "Vivo con mis padres", y Grupo 2 aglutina diversos tipos de familia extensa definida en el cuestionario mediante las siguientes respuestas: "Vivo con mi padre", "vivo con mi madre", "vivo con mis abuelos" y "vivo con otros familiares". Tal y como puede observarse en la tabla 4 hay un mayor número de sujetos que pertenecen al primero de los grupos, es decir viven en una familia nuclear.

En segundo lugar, se procedió a analizar la existencia de diferencias estadísticamente significativas según la tipología familiar y la posterior incidencia de los/as menores en situaciones de bullying. Para dicho análisis se realizó un ANOVA del 
que tal y como puede observarse en la tabla 5 se obtuvieron unos resultados que muestran esta asociación entre la tipología familiar y la participación en situaciones de bullying, concretamente en las víctimas y en los agresores.

Tabla 4. Análisis de Frecuencias de la Variable Familia

\begin{tabular}{lcc}
\hline \multicolumn{1}{c}{ Tipo familia } & Frecuencia & $\%$ \\
\hline Familia nuclear & 1525 & 90.6 \\
\hline Familia extensa & 159 & 9.4 \\
\hline Total & 1684 & 100.0 \\
\hline
\end{tabular}

Tabla 5. ANOVA Grupo familiar - Situaciones de Bullying

\begin{tabular}{llccccc}
\hline & Grupos & Suma de cuadrados & Gl & Media cuadrática & $F$ & Sig \\
\hline Sumatorio & Gr 1(1) & 350.994 & 4 & 87.748 & 9.787 & $.000^{* *}$ \\
\hline Víctimas & Gr 2(2) & 15053.673 & & 8.966 & & \\
\hline Sumatorio & Gr 1(1) & 228.363 & 4 & 57.091 & 1.607 & .170 \\
\hline Observadores & Gr 2(2) & 59657.097 & & 35.531 & & \\
\hline Sumatorio & Gr 1(1) & 200.218 & 4 & 50.054 & 2.448 & $.045^{*}$ \\
\hline Agresores & Gr 2(2) & 34333.898 & & 20.449 & & \\
\hline $\begin{array}{l}\text { (1) Grupo 1(Grupo Familia nuclear) (2) Grupo 2 (Grupo Familia no nuclear) } \\
* \text { < } \text { <.05 Intervalo de confianza 95\% ** } p<.01 \text { Intervalo de confianza 99\% }\end{array}$ & & & &
\end{tabular}

Tabla 6. Resultados Prueba $t$ de Student para las variables dependientes con muestras independientes

\begin{tabular}{lcccccc}
\hline \multirow{2}{*}{$\begin{array}{c}\text { Variables } \\
\text { Dependientes }\end{array}$} & $t$ & Gl & $\begin{array}{c}\text { Sig. } \\
\text { bilateral }\end{array}$ & $\begin{array}{c}\text { Diferencia de } \\
\text { medias }\end{array}$ & $\begin{array}{c}\text { Error típ. de la } \\
\text { diferencia }\end{array}$ & $\begin{array}{c}95 \% \text { Intervalo de } \\
\text { confianza para la } \\
\text { diferencia }\end{array}$ \\
\cline { 2 - 7 } & Inferior & Superior & Inferior & Superior & Inferior & Superior \\
\hline $\begin{array}{l}\text { Sumatorio } \\
\text { Observadores }\end{array}$ & -1.338 & 1682 & .181 & -.665 & .497 & -1.640 \\
\hline $\begin{array}{l}\text { Sumatorio } \\
\text { Víctimas }\end{array}$ & -2.179 & 1682 & .029 & -.822 & .377 & -1.561 \\
\hline $\begin{array}{l}\text { Sumatorio } \\
\text { Agresores }\end{array}$ & -2.643 & 1682 & .008 & -.665 & .252 & -1.159 \\
\hline
\end{tabular}

Finalmente, y con el fin de perseguir el mismo objetivo que con el cálculo de un ANOVA, se procedió a realizar una Prueba $t$ de Student para las variables dependientes, tal y como se refleja en la tabla 6, encontrado por un lado, que entre las familias extensas existe un mayor número de agresores/as y víctimas, y por otro lado que en ninguno de los dos grupos familiares la variable observadores cuenta con una mayor incidencia en el bullying ni en ciberbullying.

\section{DISCUSIÓN}

La familia desempeña importantes funciones durante todas las etapas vitales de la persona, pero es en la adolescencia donde ésta cobra una importancia mayor (Dekovic et al., 2004; Rodrigo et al., 2004). Por ello, en este trabajo se pretende analizar si el importante papel jugado por la familia se ve reflejado en la condiciones de violencia entre escolares, concretamente en el caso del bullying y del ciberbullying, estando éste 
último estrechamente relacionado con el avance de las nuevas redes de comunicación, en menores de Educación Secundaria.

Los resultados obtenidos en nuestro trabajo permiten afirmar que, existen más víctimas y agresores/as entre familias extensas (familias monoparentales, familias reconstituidas, crianza por parte de los abuelos,...) que entre familias nucleares. Sin embargo, a diferencia de lo que ocurre tanto con los/as agresores/as y las víctimas, no puede establecerse esta misma relación con otro de los participantes en el fenómeno del bullying como son los/as observadores/as, ya que no ha podido encontrarse una clara correspondencia entre la pertenencia a uno de los dos grupos familiares identificados y el presentar una mayor incidencia de participación en situaciones de bullying por parte de los/as observadores/as, pesar de que éstos en ocasiones son los participantes más numerosos en esta dinámica violenta (León, Felipe y Gómez, 2011).

Esta mayor incidencia en el grupo familiar no nuclear de tanto agresores/as como víctima, puede ser explicada a raíz de múltiples factores, uno de ellos puede ser los estilos educativos de las familias ya que tal y como ha sido demostrado por diferentes estudios los estilos educativos desarrollados en los diferentes tipos de familia pueden jugar un papel decisivo. Así, un gran autoritarismo en edades tempranas puede generar dificultades de interiorización de normas en los años preescolares, y una sintomatología clínica y problemas de conducta en la adolescencia (Ge, Best, Conger y Simons, 1996). Además, tal y como señala Avilés (2006) los estilos educativos y de crianza ofrecen un lugar importante para la intervención de manera que su objetivo sería dotar a sus hijos/as de herramientas psicológicas que les permitan afrontar su niñez y su adolescencia con garantías de llegar a la autonomía, la responsabilidad y a fraguarse su propia identidad.

Otro importante factor que puede ejercer una importante influencia es la comunicación familiar ya que ésta suele ser asociada con un buen ajuste psicosocial de los/as adolescentes (Musitu et al., 2001; Rodrigo et al., 2004), y por tanto dicho ajuste propiciara previsiblemente una menor incidencia en la involucración en situaciones de violencia. Además, en otra serie de factores como las actitudes de rechazo de los/as menores hacia la autoridad escolar actualmente se desconoce el grado de implicación que ejerce la familia en cuanto a la formación de dichas actitudes, aunque al respecto si se han llevado a cabo múltiples investigaciones que relacionan tanto la influencia de las actitudes positivas de los padres hacia la escuela como una mayor grado implicación en la vida académica de sus hijos/as con una mayor integración social y mejor rendimiento académico de los mismos (Martínez y Corral, 1991; Wentzel, 1998). Por ello, la potenciar la relación entre la escuela y la familia debe ser una herramienta a aplicar con el fin de reducir la violencia entre escolares, fomentando de esta manera el enfoque ecológica en todas las intervenciones relacionadas tanto con una ajustada conexión entre ambos contextos como la multitud de factores que pueden incidir en la conducta parental (Lila y Gracia, 2005). 
Por tanto, existen múltiples evidencias empíricas que constatan la importancia que tiene la familia como una variable mediadora en el desarrollo de conductas violentas entre los/as adolescentes, aunque su influencia no se produce de una forma directa, sino indirecta contando los chicos con un mayor número de agresiones directas (Cava, Musitu y Murgui, 2006). Además, tal y como ha podido ser constatado en el presente estudio existen diferencias significativas entre el pertenecer a una familia nuclear o no nuclear, y su posterior incidencia en situaciones de bullying y de ciberbullying, en dos de los diferentes grados de participación, como agresores/as y como víctimas, hecho que no ha podido ser constatado para el último de los participantes en dichas situaciones violentas, es decir para los/as observadores/as.

Los instrumentos empleados también pueden constituir una limitación en este estudio ya que se han utilizado medidas de autoinforme, las cuales suelen conllevar una serie de sesgos derivados del hecho de ser el propio sujeto el informador.

Como prospectiva cabe señalar que el estudio debería enfocarse hacia un estudio más exhaustivo de las principales características que presentas las familias nucleares y no nucleares puesto que éstas pueden llegar a influir en el desarrollo de situaciones violentas. Algunas de estas características podrían incluir aspectos relacionados con los principales estilos vinculares y educativos, la comunicación parental, las actitudes de los padres hacia la escuela,... objeto de que éstas puedan o no ser relacionadas tanto con la incidencia en las situaciones de bullying y ciberbullying como en el papel desempeñado en dichas situaciones (agresor/a, víctima, observador/a). Además el foco de estudio podría ampliarse a una posible presencia de determinados rasgos de personalidad en alguno de los dos grupos familiares identificados, y su posterior implicación en situaciones de bullying y ciberbullying, ya que estudios como los realizados por Slee y Rigby (1993) consideraron la existencia de una alta tendencia al psicoticismo en los/as agresores/as y una asociación entre victimización y neuroticismo. Por último, señalar que posiblemente existan otra serie de variables, además de la familia, que pueden estar influyendo en estas situaciones de violencia escolar tales como las relaciones existentes entre el/la menor con el profesorado y con el resto de compañeros/as, proponiéndose su análisis para estudios posteriores ya que tanto las condiciones familiares, con el importante papel que juegan en las situaciones de bullying y ciberbullying tal y como se ha descrito en el presente trabajo, como las condiciones escolares confluyen en el mantenimiento de la dinámica tanto de la agresión como de la victimización.

\section{REFERENCIAS}

Ahmad, Y. y Smith, P.K. (1990). Behavioral measures: Bullying in schools. Newsletter of Association for Child Psychology and Psychiatry, 12, 26-27. 
Avilés, J.M. (2006). Bullying: El maltrato entre iguales. Agresores, víctimas y testigos en la escuela. Salamanca: Amarú Ed.

Baurimund, D. (1991). Parenting styles and adolescent development. En J. Brooks-Gunn, R. Lerner y A.C. Peterson (Eds.), The encyclopedia of adolescence (pp. 746-758). Nueva York: Garland.

Besag, V. (1989). Bullies and Victims in Schools. Philadelphia: Open University Press.

Bowlby, J. (1969). Attachment and loss. Vol 1: Attachment. London: Hogarth Press.

Bowlby, J. (1969). El vínculo afectivo. Buenos Aires: Paidós, 1976.

Bowlby, J. (1973). La separación afectiva. Buenos Aires: Paidós, 1976.

Cava, M.J., Musitu, G. y Murgui, S. (2006). Familia y violencia escolar: el rol mediador de la autoestima y la actitud hacia la autoridad institucional. Psicothema, 18(3), 367-373.

Cerezo, F. y Esteban, M. (1992): La dinámica bully-víctima entre escolares. Diversos enfoques metodológicos. Revista de Psicología Universitas Tarraconensis, XIV, 131-145.

Defensor del Pueblo (2006). Violencia escolar: El maltrato entre iguales en la Educación Secundaria Obligatoria. Informes, estudios y documentos. Madrid.

Dekovic, M., Wissink, I.B. y Meijer, A.M. (2004). The role of family and peer relations in adolescent antisocial behaviour: comparison of four ethnic groups. Journal of Adolescence, 27, 497-514.

Derogatis, L.R. (2002). SCL-90-R. Cuestionario de 90 síntomas. Manual. Madrid: TEA Ediciones.

Floyd, N.M. (1989). Characteristics of bullies and victims in adolescent aggression. Michigan: U.M.I. Dissertation Information Service.

Ge, X., Best, K.M., Conger, R.D. y Simons, R.L. (1996). Parenting behaviors and the occurrence and co-ocurrence of adolescent depressive symptoms and conduct problems. Development Psychology, 32, 717-731.

Laslet, R. (1980). Bullies: a Children's Court in a Day School for Maldjusted Children. B.C. Journal of Special Education (Birmingam), 4, 4.

León, B., Felipe, E. y Gómez, T. (2011). Acoso escolar y Ciberbullying en centros de educación secundaria y primaria en Extremadura. Cáceres: Grupo de Investigación GIPES.

Lila, M. y Gracia, E. (2005). Determinantes de la aceptación-rechazo parental. Psicothema, 17(1), 107-111.

Lowenstein, L.F. (1977). Who is the bully? Home and School, 11, 3-4.

Martínez, J.L., Fuertes, A., Ramos, M. y Hernández, A. (2003). Substance use in adolescence: importance of parental warmth and supervision. Psicothema, 15, 161-166.

Martínez, R.A. y Corral, N. (1991). Parent and children: academic values and school achievement. International Journal of Educational Research, 15(2), 163-169.

Molpeceres, M.A., Lucas, A. y Pons, D. (2000). Experiencia escolar y orientación hacia la autoridad institucional en la adolescencia. Revista de Psicología Social, 15(2), 87-105.

Musitu, G., Buelga, S., Lila, M. y Cava, M.J. (2001). Familia y adolescencia. Madrid: Síntesis.

O'Moore, M. (1997). What do teachers need to know? En M. Elliot (Ed.), Bullying: a practical guide to coping for schools (pp. 151-166). Londres: Pitman/Kidscape.

Olweus, D. (1978). Agression in the schools: Bullies and whipping boys. Washington, D. C.: Hemisphere.

Olweus, D. (1983). Low school achievement and aggressive behavior in adolescent boys. En: D. Magnusson, y V. Allen (Eds.), Human development. An interactional perspective (pp. 353-365). Nueva York: Academic Press.

Ortega, R., Calmaestra, J. y Mora, J. (2007). Cuestionario Cyberbullying. Universidad de Córdoba, instrumento no publicado.

Ortega, R., Calmaestra, J. y Mora, J. (2008). Cyberbullying. International Journal of Psychology and Psychological Therapy, 8(2), 183-192. 
Rodrigo, M.J., Márquez, M.L., García, M., Mendoza, R., Rubio, A., Martínez, A. y Martín, J.C. (2004). Relaciones padres-hijos y estilos de vida en la adolescencia. Psicothema, 16(2), 203-210.

Slee, P. y Rigby, K. (1993). The relationship of Eysenck's personality factors and self-esteem in schoolboys. Personality and individual differences, 14(2), 371-373.

Smith, P.K., Mahdavi, J., Carvalho, C. y Tippett, N. (2006). An investigation into cyberbullying, its forms, awareness and impact, and the relationship between age and gender in cyberbullying. London: Anti-Bullying Alliance.

Wentzel, K.R. (1998). Social relationships and motivation in middle school: the role of parents, teachers and peers. Journal of Educational Psychology, 90(2), 202-209.

Recibido: 27 de febrero de 2013

Recepción Modificaciones: 8 de marzo de 2013

Aceptado: 2 de mayo de 2013 